\title{
Misrepresenting Misrepresentation
}

\author{
Michael Johnson and Ernie Lepore
}

\section{Introduction}

It's hardly news that speakers often fail to produce verbatim direct reports. Clark and his collaborators (Wade and Clark 1993, W\&C; Clark and Gerrig 1993, $\mathrm{C} \& \mathrm{G})$ attempt to exploit this widespread foible in practice to expose and undermine what they believe is a deep-seated assumption about the semantics of direct quotation, viz., that one is true just in case it is a verbatim reproduction of the original speaker's words. Accordingly, Clark denies that (1) can be true only if Joe uttered (2).

(1) Joe said 'There are cats in the garden.'

(2) There are cats in the garden.

In place of so-called wording theories, Clark defends the view that direct quotations are referring expressions that stand in a 'fundamentally different' relation to their referents than ordinary referring expressions. They are demonstrations that depict their referents.

It is surely correct that acceptable direct quotations often depart from verbatim reports. Does this establish that accurate direct quotation does not require replication? In what follows, we'll critically examine the case against wording theories and the defense of the demonstration theory. In the end, we will reject the demonstration theory, defend a sort of wording theory, and conclude that Clark and collaborators erred because they conflated reporting with quoting both of which are obviously involved in direct quotation.

\section{Case against Wording theories}

W\&C begin by distinguishing direct from indirect quotation. On most of what they say we concur, viz., that the latter can be introduced with complementizer 'that' while the former cannot; that tenses of verbs embedded inside an indirect quotation are controlled by the tense of its main verb, but not so for verbs 
embedded in direct quotation. ${ }^{1}$ Accordingly, (3) might be an accurate indirect report of an utterance of (2).

(3) Joe said that there were felines in the garden.

Based on these differences and others, most theorists conclude that what distinguishes the two is that the semantics of indirect quotation requires only 'the gist or content' of the original be preserved, while direct quotation requires 'the wording' itself be reproduced; a direct quotation is true only if words ${ }^{2}$ within its quotes were tokened by the original speaker.

$\mathrm{W} \& \mathrm{C}$ begin their critique of wording theories by observing 'a gap between theory and evidence' when it comes to attitudes about direct quotation. They say 'folk and linguistic theories appear to assume that direct quotations are verbatim reproduction' (W\&C: 805), but this assumption is 'questionable' (W\&C: 806) since speakers 'are rarely in a position to reproduce the original speaker's words verbatim, and they must know that' (W\&C: 806).

And it doesn't help, W\&C argue, to retreat to a 'weak wording theory' that has speakers acknowledging 'verbatim reproduction is unattainable' (W\&C: 806), but settling on a direct quotation being true just in case it approximates the original speaker's words (where what counts as an acceptable approximation varies with context). Weak wording theories, they claim, are unable to account for many acceptable direct quotations, such as (4)-(8) (W\&C: 806-807):

(4) You can't say, 'Well Daddy I didn't hear you'. (non-existent utterances)

(5) And I thought 'well a I'm not going to' you know 'produce any sort of functional gaffes' (unspoken thoughts)

(6) Many people have come to me and said 'Ed, why don't you run for the Senate?' (generic utterances no particular person produced)

(7) 'Well met, captain' he said, quietly, in German (translations)

(8) Senatorial hair, flaring over the eras in authoritative gray waves, says 'put me on Nightline'. (objects)

Quotations of what one can't say (4), what one thought but didn't say (5), what many have said (6), what someone said, but in a different language (7), and what senatorial hair says (8), are all of necessity not reproductions or approximations

1. We disagree, however, with their claim that the semantic values of singular terms inside indirect quotation must be determined by the context of the report, whereas those in direct quotations are invariably determined by the context of the reportee. The singular term 'I' inside 'No one said 'I'm happy' lacks a referent altogether.

2. And not its pitch or tone (if these are irrelevant to word individuation). 
of what some particular individual at some past time did say. This, W\&C take as sufficient to refute even weak wording theories. The conclusion then is that direct quotation does not require replication - exact or approximate - and so, $\mathrm{W} \& \mathrm{C}$ reject all wording theories; in their place, following $\mathrm{C} \& \mathrm{G}$, they offer a demonstration theory.

\section{Demonstrations, Non-Serious Actions, Depiction and DQ}

Key to understanding Clark's account of direct quotation is the notion of a demonstration. As C\&G use 'demonstration' it does not include demonstrating an object with a demonstrative pronoun. Rather, it's used in the ordinary sense of the word, 'to illustrate' or 'to exemplify,' the sense in which you can demonstrate (illustrate, exemplify) how someone walks, dances or utters a sentence (C\&G: 764 fn 2.; cf. also Recanati 2001: 640). ${ }^{3}$ Demonstrations enable 'others to experience what it is like to perceive the things depicted' (C\&G: 765). Alice can demonstrate for Bill how to tie one's shoes by tying her own. This allows Bill to perceive what it is like to tie one's shoes, which, combined with practice, may suffice for Bill to learn how to tie his shoes. Demonstrations, according to $\mathrm{C} \& \mathrm{G}$, differ from descriptions inasmuch as only the former are 'non-serious' actions, and depict rather than describe (C\&G: 766).

The distinction between serious and non-serious actions is from Goffman (1974): actions that are serious 'are said to be really or actually or literally occurring' (Goffman 1974: 47). An action is non-serious when it is not really or actually or literally occurring. Talking on the telephone is serious; but pretending to talk on the telephone with a banana is not.

Demonstrations, according to $C \& G$ (p. 766) are non-serious actions. $C \& G$ illustrate with Alice's demonstrating George's limp: 'she isn't,' they say, 'really or actually or literally' limping. ${ }^{4}$ Demonstrations are of what an event, state, process or object looks, sounds or feels like. In effect, 'by depicting how a thing looks, sounds or feels, they can refer to the thing itself' (C\&G: 766).

The heart of the demonstration theory is that direct quotations are demonstrations. Recanati (another demonstration theorist) outlines the view as fol-

3. There are many aspects of C\&G's account of demonstration that we will not take up here but nothing pertinent to our evaluation of their account of direct quotation will be omitted from our discussion.

4. We note in passing that being a non-serious action can't be essential to demonstration. A might really tie her shoe to demonstrate how to do so; Alice might demonstrate George's limp by actually limping; one might demonstrate fairness to one's children by literally distributing benefits to them equitably. 
lows: 'In quotation, what we demonstrate is a piece of verbal behavior - a way of speaking. We demonstrate it by producing an instance of that behavior, that is, by speaking in the relevant way' (Recanati 2001: 661). To quote, on this view, is to depict or portray a way of speaking: one's audience perceives the depiction as though (in part) they were perceiving the target of the depiction (i.e. what the depiction depicts): 'quotations are intended to give the audience an experience of what it would be like in certain respects to experience the original event' (W\&C: 808). As with other sorts of depictions, such as representational painting or sculpture, successful interpretation involves direct perceptual experience of the depiction (C\&G: 767). The referent of a quotation then - its semantic content, or what it contributes to the truth-conditions of sentences containing it - is the quotation's target, or what it depicts.

Direct quotations, say $C \& G$, selectively depict their targets. To see what this means, consider a teacher, who, after watching you bowl, sets out to demonstrate your behavior (perhaps, to help you improve your game). His actions are intended to depict your roll, but of course only selected aspects of it. It needn't matter whether you winced or smiled while bowling, or whether he uses an actual ball or whether his depiction is in slow motion, or whether he faces you while speaking. None of these 'shortcomings' belie his demonstration or depiction.

Likewise, according to the demonstration theory, with direct quotation a reporter is trying to demonstrate selected aspects of a prior speech act. In different contexts different aspects of this speech act may matter more or less, contingent on what the reporter is trying to get across to his audience: e.g. accent, grammatical or phonological infelicities, spelling, font or color of the tokened letters.

[W]hen Alice quotes George, she may depict the sentence he uttered. She can also depict his emotional state (excitement, fear, shyness), his accent (Brooklyn, Irish, Scots), his voice (raspy, nasal, whiny), and even the nonlinguistic aspects that accompanied his speech (gestures, frown, head angle) (C\&G: 769)

In regards to counter-data (4)-(8) to wording theories, W\&C say 'speakers are perfectly capable of depicting selective aspects of events that have not yet occurred, other people's thoughts, collective utterances, utterances in other languages, gestures, and much more' (W\&C: 807). So construed, in employing a direct quotation, the reporter is demonstrating/depicting, and not describing/replicating. ${ }^{5}$

5. For further discussion of the demonstration theory, see Cappelen and Lepore (2007: Ch. 8). 


\section{Experimental data contra wording theories and in support of the demonstration theory}

W\&C center their discussion around three experiments intended to test the empirical predictions of wording and demonstration theories.

In the first experiment, W\&C set out to test the accuracy predictions of wording theories. Since wording theories have it that the literal semantic content of 'A said Q' for some agent A and quotation Q, is that Q reproduces verbatim (or at least approximates) A's original utterance then, given the hypothesis that subjects, all things considered, desire to avoid saying what is literally semantically false, we should expect subjects' direct quotations to be accurate - or at least more accurate than their indirect quotations. In the experiment, 20 Stanford undergraduate students were asked to watch four videotaped scenes and subsequently to narrate the events in the scenes to a friend. The main finding of the experiment was that there was no statistically significant difference between the verbatim accuracy of direct quotations produced by the subjects as opposed to their indirect quotations. This, again with the assumption that avoiding literal falsity has lexical priority for students narrating a scene, is evidence that wording theories are themselves false.

In the second experiment, $\mathrm{W} \& \mathrm{C}$ set out 'to test the intuition that narrators do not feel compelled to quote verbatim, even when they can' (p. 814). The relevance of this hypothesis is supposed to stem again from the fact that if one assumes a wording theory, and one assumes that everyone speaks the literal truth whenever they can, no matter their aims, then one should expect that narrators do feel compelled to quote verbatim when they can, this being the literal truth (according to wording theories). W\&C found that among 16 students asked to memorize the dialogue in a scene, those 8 asked to narrate it as accurately as possible produced $99 \%$ accurate quotations, whereas the other 8 asked to entertain in their narrative (as opposed to be accurate), produced only $62 \%$ accurate quotations. We note in passing that this finding is explainable, given a wording theory, under the assumption that students who are asked to entertain may quite reasonably sacrifice the literal truth of what they say, given their instructed aim. On the other hand, if undergraduates never say what is literally false (and always avoid hyperbole, understatement, metaphor, etc.), even when the explicit aim of the conversation is entertainment, then wording theories are in trouble.

In the final experiment, 10 students listened to others narrate a dialogue, and were asked to judge the extent to which their interlocutors were accurate and to which they were attempting to be accurate. Among the 10 students, 5 thought their interlocutor was 'mostly trying to be accurate,' and the other 5 thought their interlocutor was 'trying very hard to be accurate,' but more than half of 
them thought that their interlocutor was 'not trying to get the exact wording' (p. 818). What this seems to show is that, among some of the 10 students, trying to be accurate is compatible with not trying to get the exact wording. This is problematic, say W\&C, because '[a]ccording to the wording theories...speakers should at least attempt, and be understood as attempting, to reproduce the wording of the original' (p. 816).

We are admittedly at a loss to see why this is so. A theory on which the literal semantic content of 'There are a million bugs on my windshield' is that there are a million bugs on the speaker's windshield hardly has the implication that speakers should attempt, and should be understood as attempting, to only use that sentence when it is literally true. Similarly, just because a wording theory says that 'A said Q' for agent A and quote Q is true when Q reproduces A's utterance, it doesn't follow that one ought to say that sentence only if Q reproduces A's utterance. The logic of 'is' and 'ought' is a little more complicated than that.

We do find it interesting that a handful of undergraduates consider general accuracy to be compatible with inexact wording in direct quotations. But later (Part IV, Section 2.ii), we will argue that on a more straightforward test (deference to correction) wording theories get the right result.

Even if W\&C's experiments fall short of demolishing wording theories, they go some way toward establishing as plausible the claim that when speakers directly quote, they selectively depict the original utterance. For instance, in the first experiment, W\&C found that $47 \%$ of direct quotations (as opposed to only $3 \%$ of indirect ones) were marked by intonation shifts, either at the beginning or throughout. This indicates that speakers 'dramatize' their direct reports in order to allow their audience to perceptually experience in part what the target utterance was like.

\section{Evaluating the demonstration theory}

Our criticisms of the demonstration theory divide into two sorts. First, we evaluate the claim that direct quotations are demonstrations, depictions, and nonserious actions. And then we evaluate the relevance of W\&C's experiments vis-à-vis the evaluation of wording theories. ${ }^{6}$

6. Cf. Chs. 7 and 8 of Cappellen and Lepore (2007) for other criticisms of the demonstration theory. 


\subsection{Why direct quotations are not demonstrations}

It is important to distinguish between the uncontentious claim that while directly reporting another, a speaker might also depict his utterance, ${ }^{7}$ and $C \& G$ 's stronger claim that direct quotations are depictions (viz., they are non-serious demonstrations) that depict the semantic content of a direct quotation; and so, concurrent depictions (of gestures, accent, etc.) are part of, and indistinguishable from, the semantic content of a direct quotation.

When Alice reproduces George's Irish accent, her audience experiences in part what it is like to hear George speak, recognizes Alice's intention to demonstrate George's accent, and accordingly, correctly interprets the depiction. But this is emphatically not the same thing as saying that the literal content of Alice's direct quotation is determined in any way by what she happens to be depicting. Just as you may depict someone's facial expression while referring to him (but not quoting them), you may also depict his facial expression while quoting him. The stronger semantic claim, however, is incompatible with the view that the referent of a direct quotation is not what a speaker depicts (if anything) but rather some quotable item.

Much of C\&G's evidence for treating direct quotations as depictions is analogical. Since direct quotations exhibit features that are characteristic of depictions, they conclude they are depictions. Depictions, for instance, are nonserious actions, and are selective in which aspects of what they depict that they depict. Quotations, it is argued, have all of these properties too.

\subsubsection{Quotations as non-serious actions}

C\&G (pp. 770-774) argue that direct quotations are non-serious actions. The sense in which they are deemed non-serious is that when Matt utters (9) he is not "really or actually or literally making a request" (C\&G: 770), though he is quoting one:

(9) She says, 'Well, I'd like to buy an ant.'

As already noted, demonstrations are not always (and perhaps not even typically) non-serious, but even if they were, it would still be difficult to take this line of reasoning seriously (no pun intended). Setting aside factive operators, every embedded sentence is 'non-serious' in this sense. Each of (10a-c) embeds 'I'd like to buy an ant,' yet in none is the speaker requesting to buy an ant.

7. Uncontentious, of course, insofar as one does not have general misgivings about depiction itself. 

a. She says that I'd like to buy an ant.
b. If I win the lottery, I'd like to buy an ant.
c. It might come about that I'd like to buy an ant.

Embedded requests are not 'patterned after' unembedded ones, at least in the relevant sense: they do not derive their significance from unembedded uses. Rather, their significance is derived directly by means of a compositional semantics. It's hard to see why direct quotation should be any different. In fact, it isn't.

If we ignore the irrelevant claim that the embedded sentence in (9) is not used to make a request (irrelevant because it only establishes that 'says' is not factive), we might still fruitfully ask whether Matt, in uttering (9), succeeds in discussing ants and events of buying, or whether he is using these words 'nonseriously.' Consider (11a-c) as follow-ups to (9):

(11) a. She says, 'Well, I'd like to buy an ant.' And then she found one [ant] that she liked.

b. She says, 'Well, I'd like to buy an ant.' And then she did [buy an ant].

c. She says, 'Well, I'd like to buy an ant.' And she $\operatorname{did}$ [want to buy one].

What (11a-c) suggest is that Matt, in uttering (9), talks about the state of desiring to buy an ant; about the activity of ant-buying; and about ants. If this is correct, then there's some reason to assume that in direct quotation, the quoted words are used seriously.

The present authors are divided on the question of whether the anaphora facts in $(11 \mathrm{a}-\mathrm{c})$ show that the quoted words in a direct quotation are used (in the sense of 'use' as opposed to 'mention'). But we do agree that in some broader sense of 'use' (11a-c) do show that the words are used seriously. What we mean can be seen by considering another example:

(12) Which suspects $t$ did the CIA claim they could 'interrogate $t$ without restraint'?

(13) John used to say of every woman $_{i}$ he met, 'someday I'll marry her ${ }_{i}$.'

In these contexts, the higher quantifiers ('which suspects' and 'every woman') require a variable in the lower clause that they bind. It follows then that in direct and mixed quotations, the quoted words are used seriously enough that they can meet the minimal syntactic and semantic demands of binding. Whether they are used in a stronger sense is a question beyond the scope of this paper. 
The acceptability of (11a-c) may also be exploited in a general argument against the demonstration theory, even when issues of seriousness are put to the side. Since demonstrations don't involve reference to aspects or elements of events, certain anaphoric reference to such aspects or elements after a demonstration is generally impossible, as is readily seen in (12ab):

a. You should really learn this. [George ostentatiously ties his shoe]. ??You can get one from Sally.

b. Arnold is physically impaired. [George limps around]. ??Just like Arnold did yesterday.

If one weren't merely demonstrating but were instead describing such events, the infelicitous continuations in (12ab) would be perfectly acceptable, as in (13ab):

a. You should really learn this. Watch me tie my shoe. You can get one from Sally.

b. Arnold is physically impaired. I am limping around, just like Arnold did yesterday.

It would seem to follow that utterances of direct quotations are unlike demonstrations. The internal semantic structure of a direct quotation is much like that of any other embedded clause. C\&G claim 'the internal structure of a quotation is really the structure of what is being depicted, and that can range from the raging of a person to the racket of a machine' (C\&G: 772). But this gets things precisely backwards when we consider the facts surrounding anaphora: the internal structure of the direct quotation is the structure of the objects, events and properties described by the quoted sentence.

\subsubsection{Quotations as selective depictions}

To reiterate, we are not claiming speakers never depict when they directly quote. But we are denying that utterances of direct quotations - certain bits of language spoken or written - are themselves demonstrations and that their literal semantic content - what they contribute to the propositions they semantically express is what individuals depict when they use direct quotation.

Demonstrations, recall, are alleged to be selective depictions (C\&G: 775). When Fred demonstrates a Frisbee toss, he may depict the motions involved without depicting the speed required for the toss and without letting the Frisbee go (depicting the toss's completion). Direct quotation seems similarly selective in that you may quote another in her own dialect or you may opt not to. But 
this provides no reason to infer direct quoting is selective depiction. Consider indirect speech in ordinary belief attribution. If Fred is reporting what George believes, and both Fred and his audience know George is unaware that Hesperus and Phosphorus are identical, Fred may or may not choose to use 'Hesperus' and only 'Hesperus' in reporting George's beliefs that he [George] would express using 'Hesperus.' Fred may selectively choose which aspects of George's dispositions he wants to preserve in his attributions. But none of this entails or even renders plausible the idea that standard indirect speech is depictive. Being selective need not involve being depictive. ${ }^{8}$

Second, an even deeper problem tells against direct quotations being depictions. Suppose Andrew visits the Ministry of Silly Walks. He wants to give his audience a feel for what goes on there. So he demonstrates several of the silly walks he observed on his visit. But he doesn't want his audience to think madness rules at the Ministry. So Andrew says, 'but no-one walked like this,' and then he walks in a very peculiar fashion - much too silly, even for the Ministry. Question: what did Andrew depict?

We might answer that he depicted an actual state - the state of no-one walking in a certain way. But that can't be right: the audience does not experience through Andrew's demonstration, what it's like to perceive no-one walking that way; quite the opposite! We might instead answer that he depicted a non-actual event, something that could have happened. But who is the agent of this event? Is it 'no-one'? And what is the time at which Andrew depicts the event as nonactually happening? Does his audience experience what it would be like to perceive the occurrence of a non-actual event? Can one experience that? Furthermore, which specific non-actual event, among the countless infinities of candidates, does Andrew depict? And why that one?

The right answer to our questions, of course, is that Andrew depicted nothing. A tempting position is that Andrew depicted a type of walk and not a particular event instantiating that type. We urge resistance to this temptation, for it is impossible to depict types, as Bishop Berkeley knew long ago. One can paint a man, but one cannot paint the type: man. The man one paints will have a certain height; a certain eye-color; a certain amount of hair. This is not the type: man. One can also selectively depict, and instruct one's audience to ig-

8. To be fair, $C \& G$ have many more analogies between direct quotation and selective depiction (e.g., in a depiction, the aspects selected for depiction are usually marked as such). We take it, however, that all of these analogies are equally problematic, because they are equally true of non-depictive speech, like indirect discourse (e.g., Fred may well mark his using 'Hesperus' in his reports of George to track George's dispositions, say by focusing it). 
nore aspects of one's painting as irrelevant. But this does not thereby elevate the painting to a painting of the type: man. For the instruction would have to be: ignore everything.

What this is supposed to show is that not all demonstrations are depictions. This result doesn't wreak havoc with current theories of demonstration, so far as we're aware. But it does preclude quotations from having as their semantic value what they depict. This is because it would require that perfectly sensible, truth-evaluable reports of saying, such as 'No-one said, 'It's raining," would lack a semantic value. What no-one said is not a thing, and so, it can't be depicted. ${ }^{9}$

\subsection{Building a defense of wording theories}

\subsubsection{Direct quotation and replication}

Many of the data points Clark and his collaborators adduce against wording theories (both strong and weak) hit wide of the mark. Recall (4):

$$
\text { You can't say, 'Well Daddy I didn't hear you'. }
$$

The 'problem' for wording theories is supposed to be that (4) might be true, even though there is no prior utterance that (4) reproduces, verbatim or otherwise. This however is a non sequitur. We take it that a paradigm case of a strong wording theory is one that asserts the equivalence in (14):

(14) For any direct quotation Q, 'John says Q' is true if and only if Q is a verbatim reproduction of an utterance made by the referent of 'John.'

By (14), the truth-conditions of 'You say, 'Well Daddy I didn't hear you" are that the quotation 'Well Daddy I didn't hear you' is a verbatim reproduction of an utterance made by the referent of the first occurrence of 'you' in (14). Call these truth-conditions P. Presumably then the truth conditions of a sentence composed from a negation + a weak deontic modal $+\mathrm{P}$ are that it's not the case that $\mathrm{P}$ is allowed, i.e. that it's not the case that the referent of 'you' is allowed to reproduce verbatim the quotation 'Well Daddy I didn't hear you.' This clearly does not require that anyone reproduced the quote verbatim, contra Clark et al.

9. In fairness, Recanati's version of the demonstration theory is explicit in assuming that negative cases (e.g. 'No-one said 'It's raining") don't depict anything, and the semantic value of the quote is the quote itself (that is, he assimilates such cases to flat mention). We return to this view in Part IV, Section+2.i. 
A precisely similar point can be made regarding (6):

(6) Many people have come to me and said 'Ed, why don't you run for the Senate?'

The 'problem', again, is supposed to be that the truth of (6) doesn't rest on the quotation "Ed, why don't you run for the Senate?" reproducing verbatim the utterance of any particular individual. This however is not a problem for any wording theory we're aware of. If the truth-conditions of ' $\mathrm{x}$ said $\mathrm{Q}$ ' for some direct quotation $\mathrm{Q}$ are that $\mathrm{Q}$ is a verbatim reproduction of an utterance by the object assigned to the variable ' $\mathrm{x}$,' then the truth-conditions of 'Many $\mathrm{x}$ : $\mathrm{x}$ said Q' are that $\mathrm{Q}$ is a verbatim reproduction of what many people (not any particular person) said.

The general lesson is that wording theories do not require that every direct quotation, no matter how many operators and quantifiers it is embedded under, reproduces an actual utterance. This would make a hash of directly quoting what someone might have said, or what no-one said. That wording theories lack this requirement falls out of the basic wording theoretic analysis of direct quotation (e.g. (14)) plus a compositional semantics for such words as 'not,' 'can,' 'many,' and 'no-one.'

In fact, it's somewhat ironic that Clark et al lay cases like (4) at the doorstep of wording theories, since they do create troubles for the demonstration theory. Since there is no prior utterance that the quotation "Well Daddy I didn't hear you" depicts, there's strong reason to doubt that quotation involves depiction, as we saw last section.

Other demonstration theorists haven't solved the problem either. Recanati, considering a case similar to (4) (Recanati 2001: 11), is forced to the unhappy conclusion that direct quotations embedded under negation are cases of flat mention, where the target (depicted aspect) of the quotation is the quotation itself, or properties thereof. This conclusion is unhappy first because it obviously won't work for mixed quotations (see Part $\mathrm{V}$ below) embedded under negation (a case Recanati doesn't discuss), and second, because such direct quotations share none of the hallmarks of flat mention - for example, they, unlike standard cases of flat mention, introduce discourse referents:

(15) John didn't say 'I like apples.' I know this because he hates them.

(16) The sentence 'I like apples' contains three words. ???John really hates them.

Clark's other complaints against wording theories seem equally misdirected. Consider again (8): 
Senatorial hair, flaring over the eras in authoritative gray waves, says 'put me on Nightline'

Wording theories say: it is literally false that senatorial hair says 'Put me on Nightline' because senatorial hair can't speak. Thus, if (8) is false, then wording theories actually make the right prediction. Are W\&C saying it's literally, semantically true that senatorial hair says that? If they are, then their theory is plainly false; if they're not, then the datum is irrelevant, because it fails to support the demonstration theory over any other.

What's to be said about (5), where direct quotation appears after a verb of thinking, and no prior utterance has been made?

And I thought 'Well a I'm not going to' you know 'produce any sort of functional gaffes.'

Several options present themselves, depending on how one thinks about thinking. If English speakers sometimes think in English, a strong wording theorist may plausibly assert that (5) is true if, and only if, the quotation "well a I'm not going to produce any sort of functional gaffes" is a verbatim reproduction of a prior thought had by the referent of 'I.' Alternatively, if speakers don't think in English, (5) and similar examples may be all taken for false - we, at least, don't have a clear intuition concerning truth-conditions in such cases, and are content to let theory decide. ${ }^{10}$ The important point is that $\mathrm{C} \& \mathrm{G}$ and $\mathrm{W} \& \mathrm{C}$ don't have a better view on offer. For them, quotations are depictions and as such, are supposed to enable 'others to experience what it is like to perceive the things depicted' (C\&G,: 765). But we doubt that they honestly believe the quote in (5) enables anyone to experience what it's like to perceive someone else's thoughts. It takes more than quoting a bat's thoughts to know what it's like to be a bat.

The upshot of the discussion so far is as follows: we are unimpressed by the 'problem cases' W\&C and C\&G present to motivate rejecting wording theories. None of the cases discussed so far present any problem for strong wording theories, much less for weak ones. Additionally, many of the cases (e.g. (5) and (6)), are problematic for demonstration theories, and thus, even if they motivated a rejection of wording theories, they wouldn't motivate turning to a demonstration theory. The only legitimate concern for wording theories in their discussion, as we see it, is the existence of acceptable direct quotations that are not reproductions but are rather translations of the speech of others, such as (7):

10. A third option, if agents think in language, but not in natural language, is to assimilate quoting thoughts to what we say about translation, below. 
'Well met, captain' he said, quietly, in German.

(7) is problematic for strong wording theories, because "Well met, captain" does not reproduce verbatim any German utterance; and it is a problem for weak wording theories, because it does not approximate any German utterance. If Clark et al want to limit the terminology of 'wording theories' to just these two types, then wording theories must be rejected. Our own view is that (7) is true if, and only if, the referent of 'he' said the quotation 'Well met, captain.' And we further claim you can say 'Well met, captain' in German, and you do so by ... well, saying it in German.

We should say a little more by way of motivation. Speakers permit a wide variety of divergence from verbatim reports in acceptable direct quotation. Indeed, most transcripts, which are after all just a form of direct quotation, have speakers saying fully grammatical sentences, even though, as a matter of fact, normally what issues from our mouths are incomplete ungrammatical sentences. Our utterances are usually chock-full of gaps, false starts, missing words, mistaken inflections, etc. Yet the practice of fixing up recorded interviews is ubiquitous, at least, in the press.

What this shows, we think, is that for someone to have said a quote is not a matter of that quote's reproducing a verbatim utterance of yours. If Ernie utters 'The demonstration theory ain't true,' Michael can truly report him by uttering 'Ernie said 'The demonstration theory is not true.' This is because Ernie did say that - he said it colloquially. Similarly, when we read in a history of philosophy book that Descartes said, 'I think, therefore, I am,' we don't regard the book as full of falsehoods. Descartes did say that - he said it in French. What this report shows (barring some reservations below) is something about the conditions under which a speaker can stand in a saying relation to a quoted sentence. Some acceptable direct quotations reveal to us that a speaker can stand in a saying relation to a quoted sentence (or sentence fragment) even though he never uttered a token of that sentence (or sentence fragment); he might have instead uttered only a translation of it in another language or even an ill-formed version of it in the same language.

Our view, then, is like a wording theory except we've replaced 'reproduced verbatim'/ 'approximated' in the truth conditions of direct report with 'said.' That is, for any quote Q, 'Mary said Q' is true if and only if Mary said Q; i.e. if $\mathrm{Q}$ is something Mary said; i.e. if what Mary said was Q. We doubt we can provide a fully explicit analysis of 'say' any more forthcoming than we could one for 'good.' But there's no reason to suppose saying involves depiction. It's a lot closer to verbatim reproduction, except for permitting cleaning-up and translation. It's saying. 


\subsubsection{Deference to correction}

None of the above means that anything goes. There are limits and there are errors. We permit some degree of fixing up - if we are intending to attribute a direct quotation to a speaker of an English sentence, we more likely than not will eliminate grammatical infelicities, especially if their existence is irrelevant to the purposes at hand. However reporters invariably defer to correction. If your direct quotation 'Al said, 'Mauve is my favorite color" draws the reaction 'I didn't know Al knew the word 'mauve", you might detract and defer, saying instead, 'Oh, what he really said is 'That color is my favor color' pointing at a patch of mauve. In effect, you are conceding your original report is inaccurate.

This datum dominates. Speakers invariably defer when confronted with, say, recorded documents. They might resist conceding vis-à-vis corrected infelicities (unless they matter for the discourse at hand) but they will defer (concede error) for non-verbatim reports when the original is presented. This suggests strongly wording theories are correct.

It also suggests W\&C's three experiments are irrelevant to the debate over the correct semantics of direct quotation. The fact that (W\&C: 811) 'the percentage of verbatim words for direct quotations is $38 \% \ldots$ is extraordinarily low for a theory that assumes speakers are committing themselves to verbatim reproduction' is more than balanced by a reporter's willingness to defer when confronted with inaccuracy. No one, we predict, will infer from these data that speakers don't care which words an original speaker used.

\section{General Methodological Lessons for Semantics}

W\&C seem to think that speaker behavior determines semantics. That speakers sometimes admit they are bad at verbatim direct quotation, they claim, supports their conclusion that it cannot be part of the semantics of direct quotation that it is verbatim. On the contrary, what is pertinent in determining the semantics of direct quotation is not what people do when they directly quote but rather how they respond to correction. What we find utterly relevant is that speakers invariably defer, e.g., upon re-hearing an original utterance. W\&C say, 'Speakers believe they are trying only to approximate the original speaker's utterance as best they can.' If that were right, the correct response to a charge of mistaken direct quotation would be 'we are only trying to approximate'. We sincerely doubt anyone would offer this excuse for his failure.

We end by identifying a serious deficiency in W\&C's discussion, namely, their failure to acknowledge the practice of mixed quotation (a practice we mentioned in passing in IV,2.i). The first systematic discussion of mixed quotation 
appears in Davidson (1984). Before this, the category of mixed quotation was mostly ignored. For the following twenty years, it was still relegated to footnotes. But during the last ten years or so, it has occupied front row center for anyone interested in quotation. Davidson's famous example is 'Quine said that quotation 'has a certain anomalous feature'.' This report quotes Quine by reporting what he said, but attributes to him only an utterance of the expression 'has a certain anomalous feature'. This practice is ubiquitous, but W\&C neglect the practice entirely.

There are many reasons why we might opt to mixed quote another rather than directly or indirectly quote him. We often mixed quote another because (i) the reported utterance is too long to directly quote, but the reporter wants to ensure accuracy on certain key passages, (ii) certain passages in the original utterance were particularly well put (as in, Quine says that quotation 'has a certain anomalous feature'), (iii) perhaps the words used by the original speaker were (potentially) offensive to an audience and the speaker wants to distance himself from them by indicating that they are the words of the individual being reported and not his own (as in 'Vice President Dick Cheney on the Senate floor told Senator Patrick Leahy to go 'fuck' himself'), and (iv) the expressions being mixed quoted might be ungrammatical or a solecism and the speaker might be trying to indicate that he's not responsible; or that he recognizes something is funny or odd about them (as in 'Mary said that John is a wonderful 'philtosopher' ' or 'Howard said that he 'ain't gonna take it no more"). The same sort of point extends to mixed quoting foreign expressions (as in, "Mario said that he was 'en casa' when the murder occurred').

Since W\&C, rather surprisingly, in their various experiments, do not control for mixed quotation, it's unclear what differences if any these tests could establish between direct and indirect quotation. When they conclude (W\&C: 811) that speakers are no more accurate with direct than with indirect quotation they are presuming they have identified legitimate cases of indirect quotation but their data are equally construable as mixed quotation. In fact, their method (see the 'Method' section, W\&C: 809) was to mark spoken quotations as either direct or indirect, without the option of mixed quotation. This was in accord with how they judged a 'novelist or copyeditor' would insert the punctuation; yet we submit that the results would have been substantially different, had they allowed for mixed quotation and had they punctuated as a newspaper fact-checker would have.

W\&C are testing for something; but not for semantic properties of linguistic forms. That reporters sometimes employ 'dramatized intonation...to depict annoyance, embarrassment, sarcasm' shows that they were not engaged solely in the practice of direct quotation. 


\section{Conclusion}

We argued against demonstration theorists that direct quotations are neither demonstrations nor selective depictions nor non-serious actions. We also argued that their requirement that true direct quotations depict prior speech cannot be squared with the fact that direct quotations can report what someone might have said or even what no-one said. We challenged the problematic cases they alleged against wording theories. However, a legitimate worry their discussion raises concerns direct reports of utterances translated into, or cleaned up in, the language of the direct report. These cannot be reproductions. We noted, that the saying relationship between a person and quote permits something less than a verbatim reproduction. We do not take this qualification to be an interesting compromise of wording theory, however, since the practice of invariable deference to correction is still in place. When challenged we push our direct reports further and further towards verbatim reproductions. This dominating fact about our practice of direct quotation renders, we conclude, that all of the experimental data advanced against wording theories is irrelevant. If that were not enough, we ended our discussion by complaining that Clark et al neglected to control for mixed quotation. Without some such control Clark runs the risk of contrasting apples with apples, since both direct and mixed quotation reports utilize bona fide quotation.

\section{References}

Cappelen, Herman \& Ernie Lepore, 2007 Language Turned on itself. Oxford: Oxford University Press.

Clark, Herbert H. \& Richard J. Gerrig 1990 Quotations as demonstrations. Language 66: 764-805.

Davidson, Donald 1984.

Quotation. In Inquiries into Truth and Interpretation, D. Davidson (ed.), 79-92. Oxford: Oxford University Press. (Originally published in Theory and Decision 11 (1979): 27-40.)

Goffman, Erving 1974

Frame Analysis. New York: Harper \& Row.

Malcolm, Janet 1990

The Journalist and The Murderer. New York: Vintage Books.

Recanati, Francois 2001. Open Quotation. Mind, 110(436): 637-87. 
248 Michael Johnson and Ernie Lepore

Wade, Elizabeth, \& Herbert H. Clark

1993

Reproduction and demonstration in quotations. Journal of Memory and Language 32: 805-819. 